\title{
VERY HIGH ENERGY GAMMA RAYS FROM PLERIONS : CANGAROO RESULTS
}

\author{
T.KIFUNE \\ for CANGAROO Collaboration, Institute for Cosmic Ray \\ Research, University of Tokyo, Tanashi, Tokyo 188, Japan
}

\begin{abstract}
The current status of very high energy gamma ray astronomy (in $\sim 1 \mathrm{TeV}$ region) is described by using as example results of CANGAROO (Collaboration of Australia and Nippon for a GAmma Ray Observatory in the Outback). Gamma rays at $\mathrm{TeV}$ energies, emitted through inverse Compton effect of electrons or $\pi^{0}$ decay from proton interaction, provide direct evidence on "hot" non-thermal processes of the Universe, as well as environmental features, such as the strength of magnetic field in the emission region, for the non-thermal processes.
\end{abstract}

\section{Introduction}

The number of Very High Energy Gamma Ray (VHEGR) sources is rapidly increasing from only one (Crab) in 1990 to the current value of more than five. VHEGR observation experienced a breakthrough by the Imaging Air Cerenkov Telescope (IACT; e.g. review by Cawley 1996), almost simultaneously with the launching of Compton Gamma Ray Observatory (CGRO). The $\mathrm{GeV}$ sources discovered by EGRET of CGRO are also likely VHEGR emitters and have served as a guide for VHEGR observation.

Energetic electrons (and positrons) are found abundant in point(-like) sources, plerions and Active Galactic Nuclei (AGN) to illuminate the sky bright at $\mathrm{GeV}$ and $\mathrm{TeV}$ energies (for review, Weekes et al. 1997 and references therein), while the diffuse emission from the Galactic disk is considered due to protons. As electrons radiate through inverse Compton and synchrotron processes into $\mathrm{TeV}$ band and longer wavelengths, respectively, the importance of multi-wavelengths study is built-in for VHEGR astronomy. The VHEGRs from proton progenitor of producing $\pi^{0}$ decay gamma 
rays are estimated to be of detectable fluxes from supernova remnant (SNR). Protons are abundant there and must be injected into the "accelerator" in the supernova shell (Drury et al. 1994; Naito and Takahara 1994).

\section{Galactic sources and CANGAROO results}

The CANGAROO with IACT in South Australia observes gamma rays at $\mathrm{TeV}$ energies from the southern sky, and has discovered VHEGRs from Galactic objects of plerion and SNR, while other IACTs such as the 10 $\mathrm{m}$ one of Whipple Observatory are for the northern sky, having revealed VHEGR activity of AGN such as Mrk 421 and 501.

Six gamma ray pulsars discovered by EGRET emit their largest portion of energy output into $\geq 100 \mathrm{MeV}$ as pulsed radiation which is modulated with the spin period of neutron star. The pulsed signal dissapears and only unpulsed component remains as observed at $\geq 300 \mathrm{GeV}$ energies (for review e.g. Kifune 1996 and references therein); the region of emission changes from the pulsar magnetosphere to outer region, where pulsar wind collides with the circumstellar medium. CANGAROO has detected VHEGRs from several pulsar nebulae formed in such a region, as well as from a SNR, SN 1006.

The Crab nebula is the unique case that the whole spectrum of synchrotron and inverse Compton radiation is measured, giving estimate of the magnetic field to be $\sim 270 \mu \mathrm{G}$ close to the equi-partition value (Weekes et al. 1997). The strong magnetic field and huge spin-down luminosity results in intense synchrotron radiation which are then converted to have higher energies by Compton scattering, making the Crab nebula only one unpulsed $\mathrm{GeV}$ plerion detected so far by EGRET. The energy spectrum observed can be compared in detail with model calculations (e.g. Atoyan and Aharonian 1995). The spectrum at higher energies is more sensitive to emission models, because of the effects of the maximum energy of accelerated electrons and/or Klein-Nishina fall-off of the electron-photon scattering cross section in inverse Compton process. The CANGAROO observation of the Crab, thanks to good sensitivity at $\geq 10 \mathrm{TeV}$ enabled by the observation at large zenith angles $\left(53^{\circ}-60^{\circ}\right)$ from the observation site in the southern hemisphere, has reported a constant power index of energy spectrum up to at least $50 \mathrm{TeV}$ and possibly to $\sim 100 \mathrm{TeV}$ (Tanimori et al. 1997).

Following the results on PSR B1706-44 (Kifune et al. 1995), VHEGRs from Vela pulsar direction was found. The emission region is displaced by about $0.13^{\circ}$ from the pulsar, apparently in accordance with the birth place of the Vela pulsar (Yoshikoshi et al. 1997). The ratio $\eta=\mathrm{L}_{\mathrm{s}} / \mathrm{L}_{\text {ic }}$ of luminosity in X-ray synchrotron radiation to inverse Compton is equal to the ratio $\mathrm{W}_{\mathrm{mag}} / \mathrm{W}_{2.7 \mathrm{~K}}$ of energy density of magnetic field to that of ambient taget photons for inverse Compton scattering. For Vela and PSR B1706-44, 


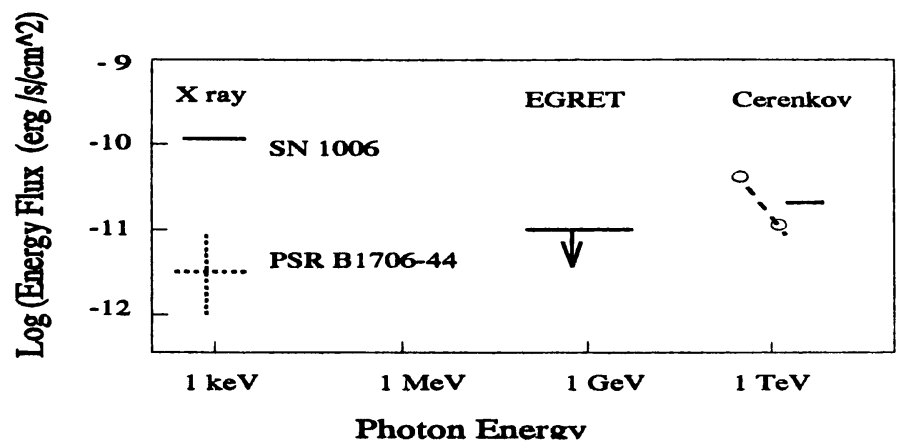

Figure 1. Comparison of fluence to X-ray and $\mathrm{GeV}$ band.

magnetic field $\mathrm{B}$ in the nebula is estimated as weak as that in the interstellar medium, $\sim 3 \mu \mathrm{G}$. The synchrotron life time of electrons calculated from the magnetic field allows the electrons generated at the birth time of Vela pulsar to survive until the present time (Harding et al. 1997). If $\mathrm{B}$ is $\sim 3 \mu \mathrm{G}$ in the pulsar nebulae of PSR B1706-44 and Vela, the energy density of progenitor electrons is higher, by an order of magnitude, than $B / 8 \pi^{2}$. The two energy densities are not in equi-partition.

However, the VHEGR data can imply a higher B with a constraint on life time and spatial confinement of electrons. By deviding the nebula into two region, then $\eta=\frac{1}{1+A} \cdot\left(\eta_{2}+A \cdot \eta_{1}\right)$, where $\mathrm{A}=\tau_{\mathrm{e}} / \tau_{2}$ is the ratio of escape time $\tau_{\mathrm{e}}$ of progenitor electrons (out of the central region 1 of stronger $\mathrm{B}$ ) to the total life time $\tau_{2}$ of electrons (in the peripheral region 2). When $\mathrm{A}$ $\ll 1, \eta$ approaches to $\eta_{2}$. In the case of PSR B1706-44, $\mathrm{B}_{1} \sim 20 \mu \mathrm{G}$ can be compatible with both of the CANGAROO and X-ray data, with the escape time $\tau_{\mathrm{e}} \sim 10 \cdot(\mathrm{E} / 20 \mathrm{TeV})^{-\delta}$ yrs and $\delta=0 \sim 0.5$ (Aharonian et al. 1997).

\section{Discussions}

The sensitivity of IACT is at a level somewhat below $10^{-11} \mathrm{erg} \mathrm{s}^{-1} \mathrm{~cm}^{-2}$, which corresponds to the luminosity $10^{33} \mathrm{erg} \mathrm{s}^{-1}$ at a distance of $1 \mathrm{kpc}$. In Fig. 1, observed fluence is compared between X-ray, GeV and TeV gamma rays for SN 1006 (solid line) and PSR B1706-44 (dashed line). The upper limit in $\mathrm{GeV}$ region is consistent with a spectrum having two peaks in X-ray and $\mathrm{TeV}$ band, each peaked one corresponding respectively to synchrotron and inverse Compton radiation. The spectrum implies abundant production and acceleration of electrons and positrons with a condition of $B$ not much greater than $\sim 10 \mu \mathrm{G}$. The comparison of the two bands of $\mathrm{GeV}$ and $\mathrm{TeV}$ can be consistent with a spectrum of power index flatter than -2 , which 
proton progenitor by shock acceleration can hardly explain. In order to know an allowed possible fraction of contribution from $\pi^{0}$ decay gamma rays due to protons, the energy spectrum of better accuracies is required through $\mathrm{GeV}$ to $\mathrm{TeV}$ bands.

The SNRs associated with unidentified EGRET sources attract interest of VHEGR observations, because the shock acceleration theory predicts detectable $\mathrm{TeV}$ fluxes when we attribute the EGRET flux to $\pi^{0}$ decay gamma rays from protons that are accelerated in the SNR shell and then collide with the matter of enhanced density in the nearby molecular cloud. The efforts are so far not successful to detect VHEGRs (Lessart et al. 1997; Hess et al. 1997). However, the complex features associated with those SNRs leave room for a variety of mechanisms other than $\pi^{0}$ decay process to explain the $\mathrm{GeV}$ emission and no detection at $\mathrm{TeV}$. On the other hand, SN 1006 is of comparatively simple, "pure" shell type SNR, and the CANGAROO result has shown that particles with Lorentz factor $\gamma \geq 10^{8}$ undoubtedly exist as suggested from non-thermal X-rays (Koyama et al. 1995).

A step is forwarded towards the "origin of cosmic rays" through the study of VHEGRs. The progress will also provide us with the means to understand wealth of phenomena like the complex structure of SNRs. The Vela, PSR B1706-44 nebulae and SN 1006 appear brighter at TeV energies than in $\mathrm{GeV}$ region of EGRET. There is some indication of detection (Aharonian and Heinzelmann 1997 on micro quasar GRS 1915+105; Turver et al. 1997 on Cen X-3) that suggests new types of time-variable sources, encouraging further watching on the VHEGR sky.

\section{References}

Aharonian, F.A., Atoyan, A.M., and Kifune, T. (1997) MNRAS, Vol. 291, 162

Aharonian, F.A. and Heinzelmann, G. (1997) Nucl. Phys. B, to be published (astro$\mathrm{ph} / 9702059)$

Atoyan, A.M. and Aharonian, F.A. (1996) MNRAS, Vol. 278, 525

Cawley, M.F. (1996) Nuovo Cimento, Vol. 19C, pp. 959-962

Drury, L.O'C., Aharonian, F.A., and Völk, H.J. (1994) Astron. Ap. Vol. 287959

Harding, A.K., De Jager, O.C., and Gotthelf, E. (1997) Proc. 25th ICRC (Durban) Vol. 3 325

Hess, M. et al. (1997) Proc. 25th ICRC (Durban) Vol. 3229

Kifune, T. et al. (1995) Ap J Lett. Vol 438, L91

Kifune, T. (1996) in Pulsars: Problems and Progress, IA U Coll. 160, pp. 339-346

Koyama, K. et al. (1995) Nature Vol 378, 255

Lessard, R.W. et al. (1997) Ṕroc. 25th ICRC (Durban) Vol. 3233

Naito, T. and Takahara, F. (1994) J. Phys. G.: Nucl. Part. Phys. Vol. 20477

Tanimori, T. et al. (1997) to be published in $A p J$ Lett.

Turver, K.E. et al. (1997) to appear in Proc. Kruger Workshop "Towards major atmospheric Čerenkov Telescope $V$

Weekes, T.C., Aharonian, F.A., Fegan, D.J., and Kifune, T. (1997), to appear in Proc. of the 4th Compton Symposium

Yoshikoshi, T. et al. (1997) Ap J Lett. Vol 487, L95 